\title{
Bismuth Toxicity: A Rare Cause of Neurologic Dysfunction
}

\author{
Paul T. Reynolds ${ }^{1}$, Kathleen C. Abalos ${ }^{1}$, Jennifer Hopp ${ }^{2}$, Mark E. Williams ${ }^{1}$ \\ ${ }^{1}$ University of Virginia, Charlottesville, USA; ${ }^{2}$ Hospital of the University of Pennsylvania, Philadelphia, USA. \\ Email: paul.t.reynolds@gmail.com
}

Received October $14^{\text {th }}, 2011$; revised November $30^{\text {th }}, 2011$; accepted December $25^{\text {th }}, 2011$

\begin{abstract}
Bismuth subsalicylate (Pepto-Bismol ${ }^{\circledR}$ ) and other bismuth-containing compounds have been used for many years to treat gastroenterological complaints. Although safe in the majority of patients, bismuth can cause a well-described toxic state marked by progressive neurological decline. Features of bismuth toxicity include confusion, postural instability, myoclonus, and problems with language [1]. This presentation can masquerade as other causes of progressive neurologic dysfunction including Creutzfeld-Jakob Disease (CJD), Hashimoto's Encephalopathy, and others. In this case study, we present a patient who was using bismuth salicylate in toxic quantities to help control diarrhea. On initial presentation, several diagnoses were entertained before bismuth levels were obtained. This case study highlights the fact that bismuth toxicity, while rare, should be considered in a patient with progressive neurological decline. Also, we hope this case reminds physicians of a severe consequence of a common, readily available medication.
\end{abstract}

Keywords: Bismuth Toxicity; Creutzfeld-Jakob

\section{Case Report}

A 56 year old woman who had recently begun treatment for collagenous colitis presented to her local hospital with several days of psychomotor retardation, decreased concentration, tremor of her hands, visual hallucinations, and postural instability preventing her from standing on her own. The patient was concurrently being treated on stable doses of medications for irritable bowel syndrome, hypertension, hypothyroidism, and depression.

Her husband stated that her concentration had been gradually decreasing for the previous two weeks but had profoundly worsened in the last couple days. There is no record that the patient saw a clinician for mental status deterioration prior to her hospital presentation. During her stay at the hospital, the patient became more delirious and somnolent and began to experience myoclonic jerks and hyperreflexia. Serotonin syndrome was considered, and the patient's serotonergic medications (escitalopram, duloxetine) were held. The patient's lack of improvement over the next three days resulted in transfer to a teaching hospital, where she was admitted to the Neurology Service.

Initial physical exam showed temperature 37.0 degrees Celsius, blood pressure 165/94 mm Hg, pulse 117, respirations 16 , oxygen saturations $98 \%$ on room air. The patient provided one word answers to questions, was lethargic with impaired attention, and could express orientation correctly only to person. Cranial nerves II-XI were intact, but patient could not protrude tongue. Muscle bulk and strength were normal, but tone was markedly rigid throughout. She demonstrated frequent myoclonic jerks and occasionally exhibited intention tremor. Reflex examination demonstrated hyperactive spreading reflexes at all stations with upgoing toes bilaterally. All other aspects of the physical exam were unremarkable. Laboratory tests, including thyroid function tests, B12 levels, $\mathrm{RPR}$, and various microbiological assays were within normal limits. A noncontrast head MRI and intracranial/ neck MRA obtained on admission to UVA demonstrated no acute process or mass effect. Lumbar puncture showed a slightly elevated white blood cell count (7 and 11/UL in two tubes from same tap; $95 \%$ lymphocytes) but no other abnormalities. An EEG on admission revealed moderate, but nonspecific, encephalopathy. The results of a paraneoplastic autoantibody panel were normal.

A toxicology consultation was ordered to assess the possible contribution of a recently started homeopathic medication (black cohosh) to the patient's presentation, but this service was more intrigued with the idea of bismuth toxicity. Two months earlier, the patient had been started on $45 \mathrm{~mL}$ three times daily $(262 \mathrm{mg} / 15 \mathrm{~mL})$ of bismuth subsalicylate to help control the diarrheal symptoms of her collagenous colitis. According to the patient's husband, she had been increasing her use of this medication over the past few weeks to titrate to her symptom level.

The bismuth level in the patient's blood was found to be $397.3 \mathrm{ng} / \mathrm{mL}$, which was well above the testing labo- 
ratory's $200.0 \mathrm{ng} / \mathrm{mL}$ threshold for bismuth toxicity. Bismuth levels in the urine were $292.5 \mathrm{ng} / \mathrm{mL}$, where a normal value should be less than $1.0 \mathrm{ng} / \mathrm{mL}$.

Her bismuth subsalicylate was held, and over the next 2 days the patient became more alert, had decreased myoclonus, and exhibited less muscular rigidity. The patient was discharged to a rehabilitation facility, where her functional and mental status continued to improve. The patient was seen several months later for a clinic visit to assess her collagenous colitis, and her mental status was reported as normal.

\section{Discussion}

Bismuth encephalopathy is a rare syndrome that can mimic other neurologic conditions. It presents as a remarkably consistent syndrome (Table 1), with four signs being present: severe confusion, myoclonus, lack of coordination with standing or walking (astasia-abasia), and problems with language [1]. Our patient exhibited all of these characteristics. Dysarthria has also been described in the literature, [2] and bismuth toxicity in animal models has been shown to destroy testicular macrophages, which leads to lower testosterone levels and reproductive dysfunction [3].

Ninety-nine percent of ingested bismuth is not absorbed by the human body and passes unaltered through the feces. The small percent that is absorbed into the body through the GI tract is distributed throughout all the body's tissues with slightly higher concentrations in the liver and kidneys. Absorbed bismuth is not metabolized, and is eliminated from the body via renal or hepatic processes [4].

Severity of symptoms coordinates with the level of bismuth found in the serum [5]. Normal serum bismuth levels are 10 - $50 \mathrm{ug} / \mathrm{L}$; toxicity rarely occurs if the serum concentration is below $50 \mathrm{ug} / \mathrm{L}$ [4]. Initially, there is a prodromal phase with alterations of mood and sleep, specifically insomnia, lethargy, apathy, malaise, anxiety and irritability. These symptoms are noted first usually by spouses and family members. This phase can last for weeks or months as bismuth levels continue to rise. At some point, there is a rapid escalation in symptoms and encephalopathy occurs. Neurologic symptoms worsen

Table 1. Signs of bismuth encephalopathy $[1,2,5]$.

\begin{tabular}{ll}
\hline \multicolumn{1}{c}{ Signs of Bismuth Encephalopathy } \\
\hline • & \multicolumn{1}{c}{ Severe confusion gradually developing over weeks but } \\
markedly worsening over $24-48$ hour period; can lead to \\
coma \\
- Myoclonus \\
Lack of coordination with standing or walking (astasia- \\
- $\begin{array}{l}\text { abasia) } \\
\text { - }\end{array}$ \\
\hline
\end{tabular}

markedly in a 24 - 48 hour period and include instability, ataxia, confusion, dysarthria, hallucinations, parasthesias, seizures, and myoclonic jerks. Somnolence and coma can occur [5]. Reliable mortality data is unavailable, most likely due to the rareness of the condition.

Some of the conditions that may be in the differential diagnosis for bismuth toxicity are CJD, Hashimoto's encephalopathy, progressive myoclonic ataxia. and serotonin syndrome. Although the presentations may be similar, there are several differences between these disorders (Table 2). CJD can only be definitively diagnosed on autopsy, but sensitive tests such as detection of periodic sharp waves on electroencephalogram (EEG) and 14-3-3 proteins in the CSF can often lead to a presumptive diagnosis of CJD [6]. Our patient's lumbar puncture and EEG were inconsistent with CJD. Hashimoto's encephalopathy, like CJD and bismuth toxicity, is rare but often presents with clues such as a degree of thyroid dysfunction (although euthyroid presentation is possible) and antithyroid autoantibodies [7,8]. Progressive myoclonic ataxia, which can be caused by celiac disease, has myoclonus and ataxia as its primary features, but usually does not present with cognitive decline $[9,10]$. Serotonin syndrome was briefly considered in our patient due to her medications and the presence of myoclonus, but serotonin syndrome usually presents with more agitation and features of autonomic dysfunction such as fever and hyperhidrosis [11]. Further, the patient did not improve by discontinuing serotonergic medications during her hospital stay.

One of the reasons that bismuth toxicity is often hard to diagnose is because of its low incidence. In one meta-analysis of patients taking bismuth compounds to eradicate $H$. pylori, no adverse neurologic effects were recorded across 35 randomly controlled trials, including 11 studies in which the patient took the bismuth compound for greater than one month [12]. In fact, the only side effect that was statistically significant in this analysis was increased frequency of darkened stools. Blackening of the tongue has been reported in the literature as a harmless side effect of bismuth toxicity, but this is rare [13].

Table 2. Major clues differentiating bismuth toxicity from other disorders [6-10].

\begin{tabular}{|c|c|}
\hline Disorder & $\begin{array}{c}\text { Commonly Seen Feature Inconsistent } \\
\text { with Bismuth Toxicity }\end{array}$ \\
\hline Creutzfeld-Jakob & - $\quad$ Periodic sharp waves on EEG \\
\hline Disease & - $\quad$ Presence of 14-3-3 proteins in CSF \\
\hline Hashimoto’s & - Thyroid dysfunction \\
\hline Encephalopathy & - $\quad$ Presence of antithyroid autoantibodies \\
\hline Progressive & Absence of Cơnitive decline \\
\hline Myoclonic Ataxia & 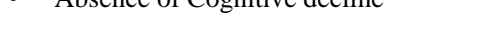 \\
\hline Serotonin & - Agitation \\
\hline Syndrome & - $\quad$ Pronounced Dysautonomia \\
\hline
\end{tabular}


Despite the low side effect profile of bismuth compounds, bismuth toxicity can be devastating when it occurs.

Since the mechanism of bismuth toxicity has not been well described, it is difficult to predict who will be affected by it and what kinds of treatment will work best. The primary treatment is removal of the bismuth compound from the patient's intake. By removing these substances, bismuth encephalopathy generally has been demonstrated to improve gradually over weeks to months with only supportive care [2,14]. Our patient returned four months after discharge without neurologic complaints.

The role of chelators such as D,L-2,3-dimercaptopropane-1-sulfonic acid (DMPS) as a remedy is unclear. One case report described clinical deterioration with DMPS and eventual discontinuation of the chelator, [2] while another reported recovery with this therapy [15]. The efficacy of DMPS has not been clearly demonstrated. Dimercaprol (BAL) in doses of $3 \mathrm{mg} / \mathrm{kg}$ may be tried within 8 to 12 hours of a bismuth poisoning. Later administration may be ineffective due to rapid absorption. One study has shown BAL to be ineffective in humans. When 9 different chelators were tested in animals, the most effective commonly available agent was D-penicillamine. Penicillamine has not been used in human bismuth overdoses [4]. Our patient recovered without chelation therapy.

Bismuth encephalopathy can result from long-term ingestion of bismuth subsalicylate. Due to the common use of bismuth subsalicylate and its usual absence of side effects, bismuth toxicity can be easily forgotten as a cause of neurologic dysfunction. This case report demonstrates that the toxicity, while rare, should be considered when a clinician is suspecting similarly presenting disorders such as CJD, Hashimoto's Encephalopathy, and Progressive Myoclonic Ataxia.

Treatment of bismuth encephalopathy consists primarily of discontinuation of the offending agent along with supportive care.

\section{Acknowledgements}

The authors wish to thank Dr. Christopher Holstege for his assistance with this manuscript.

\section{REFERENCES}

[1] P. Loiseau, P. Henry, P. Jallon and M. Legroux, "Iatrogenic Myoclonic Encephalopathies Caused by Bismuth Salts," Journal of the Neurological Sciences, Vol. 27, No. 2, 1976, pp. 133-143. doi:10.1016/0022-510X(76)90056-3

[2] M. Teepker, H. M. Hamer, S. Knake, O. Bandmann, W. H. Oertel and F. Rosenow, "Myoclonic Encephalopathy Caused by Chronic Bismuth Abuse,” Epileptic Disorders,
Vol. 4, No. 4, 2002, pp. 229-233.

[3] J. C. Hutson, "Effects of Bismuth Citrate on the Viability and Function of Leydig Cells and Testicular Macrophages," Journal of Applied Toxicology, Vol. 25, No. 3, 2005, pp. 234-238. doi:10.1002/jat.1060

[4] Micromedex ${ }^{\circledR}$, “Healthcare Series," Thomson Reuters (Healthcare) Inc., Greenwood Village, Accessed 21 December 2011.

[5] P. M. LeQuesne, “Toxic Substances and the Nervous System: The Role of Clinical Observation,” Journal of Neurology, Neurosurgery, and Psychiatry, Vol. 44, No. 1, 1981, pp. 1-8. doi:10.1136/jnnp.44.1.1

[6] I. Zerr, M. Pocchiari, S. Collins, et al., "Analysis of EEG and CSF 14-3-3 Proteins as Aids to the Diagnosis of Creutzfeldt-Jakob Disease,” Neurology, Vol. 55, No. 6, 2000, pp. 811-815.

[7] I. Kothbauer-Margreiter, M. Sturzenegger, J. Komor, R. Baumgartner and C. W. Hess, "Encephalopathy Associated with Hashimoto Thyroiditis: Diagnosis and Treatment," Journal of Neurology, Vol. 243, No. 8, 1996, pp. 585-593. doi:10.1007/BF00900946

[8] F. Ferracci, G. Bertiato and G. Moretto, “Hashimoto’s Encephalopathy: Epidemiologic Data and Pathogenetic Considerations,” Journal of the Neurological Sciences, Vol. 217, No. 2, 2004, pp. 165-168. doi:10.1016/j.jns.2003.09.007

[9] M. Borg, “Symptomatic Myoclonus,” Neurophysiologie Clinique, Vol. 36, No. 5-6, 2006, pp. 309-318. doi:10.1016/j.neucli.2006.12.006

[10] C. D. Marsden, A. E. Harding, J. A. Obeso and C. S. Lu, "Progressive Myoclonic Ataxia (the Ramsay Hunt Syndrome)," Archives of Neurology, Vol. 47, No. 10, 1990, pp. 1121-1125. doi:10.1001/archneur.1990.00530100091019

[11] P. Birmes, D. Coppin, L. Schmitt and D. Lauque, "Serotonin Syndrome: A Brief Review,” Canadian Medical Association Journal, Vol. 168, No. 11, 2003, pp. 14391442.

[12] A. C. Ford, P. Malfertheiner, M. Giguere, J. Santana, M. Khan and P. Moayyedi, "Adverse Events with Bismuth Salts for Helicobacter Pylori Eradication: Systematic Review and Meta-Analysis,” World Journal of Gastroenterology, Vol. 14, No. 48, 2008, pp. 7361-7370. doi:10.3748/wjg.14.7361

[13] M. D. Ioffreda, C. A. Gordon, D. R. Adams, S. J. Naides and J. J. Miller, "Black Tongue," Archives of Dermatology, Vol. 137, No. 7, 2001, pp. 968-969.

[14] M. F. Gordon, R. I. Abrams, D. B. Rubin, W. B. Barr and D. D. Correa, "Bismuth Subsalicylate Toxicity as a Cause of Prolonged Encephalopathy with Myoclonus," Movement Disorders, Vol. 10, No. 2, 1995, pp. 220-222. doi:10.1002/mds.870100215

[15] H. Ovaska, D. M. Wood, I. House, P. I. Dargan, A. L. Jones and S. Murray, "Severe Iatrogenic Bismuth Poisoning with BIsmuth Iodoform Paraffin Paste Treated with DMPS Chelation,” Clinical Toxicology, Vol. 46, No. 9, 2008, pp. 855-857. doi:10.1080/15563650801953182 